\title{
A perspectiva dos profissionais da Atenção Primária à Saúde sobre o apoio matricial em saúde mental
}

\author{
The perspective of professionals in primary health care \\ on matrix support in mental health
}

Programa de PósGraduação em Promoção da Saúde, Universidade Luterana do Brasil. Av. Farroupilha 8001, São José 92425-900 Canoas RS Brasil. 92425-900 alicehirdes@gmail.com

\begin{abstract}
The research investigates the matrix support in mental health in Primary Health Care in order to identify the guidelines, professional principles and values that permeate the process. The study used a qualitative approach with triangulation of techniques, and was performed with professionals from the Family Health Strategy. The results reveal a work process which is based on personalized relations, guided by the broadening of clinical practice and mediated by principles, such as inter-disciplinarity, bonding, comprehensiveness, accessibility, co-responsibilization and longitudinality. The values that underpin the relationship with the specialist are organized around the bond, trust and honesty. Difficulties in taking patients to Psychosocial Care Centers arise as obstacles to networking. The bond with the specialist, the structure of Family Health teams and the exchange of experiences and knowledge are identified as facilitating factors. The conclusions drawn are that the networking and the systematic and longitudinal support by specialists are key to decentralization in mental health.
\end{abstract}

Key words Mental health, Decentralization, Primary health care, Matrix support, Organizational innovation
Resumo A pesquisa investiga o apoio matricial em saúde mental na Atenção Primária à Saúde com vista à identificação de diretrizes, princípios profissionais e valores que permeiam o processo. $O$ estudo teve uma abordagem qualitativa, com triangulação de técnicas, e foi realizado com profissionais da Estratégia Saúde da Família. Os resultados denotam um processo de trabalho sustentado por relações personalizadas, pautado pela ampliação da clínica, mediado por princípios como a interdisciplinaridade, o vínculo, a integralidade, a acessibilidade, a corresponsabilização e longitudinalidade. Os valores que sustentam a relação com o especialista organizam-se em torno do vínculo, da confiança e da honestidade. As dificuldades de encaminhamento ao Centro de Atenção Psicossocial aparecem como barreiras à articulação em rede. O vínculo com o especialista, a estrutura das equipes de Saúde da Família e a troca de experiências e saberes são identificados como fatores facilitadores. Conclui-se que a articulação em rede e o suporte sistemático e longitudinal de especialistas são fundamentais para a descentralização em saúde mental.

Palavras-chave Saúde mental, Descentralização, Atenção Primária à Saúde, Apoio matricial, Inovação organizacional 


\section{Introdução}

A recente aprovação do Plano de Ação Global de Saúde Mental 2013-2020¹, pela Assembleia Geral da Organização Mundial de Saúde (OMS), visibiliza a relevância da proteção e promoção dos direitos humanos de portadores de transtornos mentais e do cuidado de base comunitária. Outro documento formaliza que pessoas com transtorno mental podem ser consideradas populações vulneráveis ${ }^{2}$, e são notórios os esforços de inclusão desses transtornos na agenda de Doenças Crônicas Não Transmissíveis ${ }^{3}$.

Às razões dessa integração sobrepõe-se a lacuna de tratamento para pessoas com transtornos mentais, que excede os $50 \%$ em todos os países do mundo e aproxima-se de $90 \%$ em países com recursos escassos. As estratégias para reduzir essa lacuna podem ser alcançadas por meio de três elementos: o compartilhamento de tarefas com profissionais não especialistas; o aumento dos recursos dos serviços especializados para fornecer supervisão e apoio eficaz e sustentado; e, a descentralização desses recursos ${ }^{4}$.

$\mathrm{Na}$ América Latina, a experiência chilena de integração da saúde mental na Atenção Primária à Saúde (APS) mostra que as estratégias devem contemplar a priorização de problemas de expressiva prevalência e incapacidade, a definição de atividades para a atenção primária e secundária; o nível de resolução da APS; a consultoria em saúde mental e o cálculo de recursos necessários para a saúde mental na APS. Nessa integração são críticos os mecanismos de articulação e de apoio entre profissionais da atenção secundária e primária ${ }^{5}$. É esse o objeto da presente pesquisa.

No Brasil, os estudos de Onocko Campos et al. ${ }^{6}$ indicaram que o apoio matricial se mostrou potente para definir fluxos, qualificar as equipes e promover assistência conjunta e compartilhada. Entretanto, há necessidade de investimento no preparo dos profissionais e nos processos de transformação das práticas. Os autores apontam paradigmas em confronto - o biomédico e o da ampliação da clínica. Para Campos ${ }^{7}$ os processos de matriciamento devem promover relações horizontais. Assim, o apoiador e a equipe de referência podem trabalhar na construção de visões multi e transdisciplinares, compartilhando saberes de seu núcleo de conhecimento e incorporando demandas de outros profissionais e da comunidade.

A descentralização em saúde mental, por meio do Apoio Matricial (AM) na APS, pode configurar-se como ferramenta para o trabalho. Essa metodologia articula-se aos princípios do
Sistema Único de Saúde (SUS) ao contemplar o acesso universal, a integralidade e a equidade. Encontra ressonância, ainda, na Reforma Psiquiátrica brasileira e no oitavo ciclo de desenvolvimento da APS no Brasil ${ }^{8}$ que tem como foco a consolidação da Estratégia Saúde da Família (ESF). Atende, também a necessidade de implementação dos Núcleos de Saúde da Família (NASF), ainda que, em alguns municípios, o AM tenha sido instituído e precedido a Portaria ${ }^{9}$ que institui os NASF.

Os campos de interação saúde mental e APS, mediante o envolvimento de especialistas e generalistas, promovem novas relações, interações e práticas profissionais. Poderão, também, promover um novo paradigma de saúde pública, que incorpore efetivamente a noção da integralidade do cuidado. Desse modo, objetivou-se investigar o AM em saúde mental na APS, na perspectiva dos profissionais generalistas, com vista à identificação das diretrizes, princípios e valores profissionais que permeiam o processo.

\section{Metodologia}

Trata-se de uma pesquisa descritivo-analítica, com abordagem qualitativa, realizada em município da região metropolitana de Porto Alegre, RS, Brasil. O referido município possui o sexto maior PIB e a sexta maior população do estado, com cerca de 270.763 habitantes. Sua economia é voltada para a indústria, com destaque para o polo automotivo, o comércio e a prestação de serviços. Em 2006, habilitou-se para o processo de gestão plena da atenção à saúde. Desde 2007, suas doze Unidades de Saúde da Família (USF) são acompanhadas sistematicamente pelas equipes matriciais de saúde mental e, mais recentemente, os catorze postos de saúde que operam no modelo tradicional (UBS) também passaram a ter essa metodologia instituída, num total de vinte e seis Unidades de Saúde (USF e UBS). A rede secundária contempla três Centros de Atenção Psicossocial (CAPS): o CAPS II, o CAPSad e o CAPSi. Na atenção terciária, há a retaguarda de um serviço de emergência com leitos psiquiátricos, além dos de internação de saúde mental no hospital geral. A descentralização em saúde mental foi precedida pela realização de grupos na comunidade. Posteriormente, o AM foi incorporado como uma filosofia de trabalho pelos especialistas, mantendo-se as duas frentes de trabalho. A implementação do AM no município antecedeu a Portaria 154 de 2008, que instituiu os NASF?. 
Os participantes da pesquisa foram profissionais envolvidos no AM, quais sejam: profissionais de saúde mental que atuam como apoiadores matriciais (AM); profissionais das equipes de referência das USF; profissionais das UBS tradicionais. No total foram entrevistados 21 participantes e realizados três grupos focais (GF). O recorte aqui apresentado refere-se ao grupo dos profissionais das equipes de referência das USF envolvidos no AM, totalizando oito sujeitos. Os critérios de inclusão referiam-se à atuação em USF que contam com o apoio matricial (AM) em Saúde Mental há pelo menos dois anos e à participação sistemática nas reuniões da equipe matricial. Foi realizado contato prévio com os serviços e os sujeitos entrevistados, observando os critérios de amostragem previamente estabelecidos. Primeiramente foram realizadas as entrevistas semiestruturadas e após, o GF. Para ambas as técnicas, os participantes foram agendados previamente, em concordância com o gestor/ coordenador do serviço. Destaca-se a inclusão de profissionais atuantes nas zonas rural e urbana.

Os instrumentos utilizados foram entrevistas semiestruturadas e grupos focais $(\mathrm{GF})^{10,11}$. Com eles se buscou conhecer os processos, as atitudes e os mecanismos envolvidos no sentido de desvendar o que os participantes pensam, mas, sobretudo, por que os participantes pensam como pensam ${ }^{10}$. Com vista a aprofundar a discussão e trazer as diferentes perspectivas em jogo, os participantes dos GF foram agrupados em especialistas (AM); profissionais das USF e profissionais das UBS tradicionais. Esse agrupamento teve por objetivo, também, maximizar o potencial de comparação inter e intragrupos. Entretanto, para além da ideia de produzir dados que possam ser comparados para confirmar ou contradizer os resultados dos dois métodos (entrevistas e GF), a triangulação de métodos na pesquisa poderá fornecer insights adicionais e dados analíticos que poderão ser utilizados como um recurso para produzir bases de dados paralelas ${ }^{11}$. Tanto para as entrevistas como para os GF utilizou-se um roteiro. Os dados foram coletados no período compreendido entre abril e setembro de 2013.

Em relação aos procedimentos analíticos das entrevistas semiestruturadas e GF, foi utilizada a análise de conteúdo, na modalidade temática, proposta por Minayo ${ }^{10}$ que compreende: a ordenação, a classificação e a análise final dos dados. A ordenação dos dados consistiu na transcrição das entrevistas; na releitura do material; na organização dos relatos em determinada ordem, de acordo com a proposta analítica. Foram criados vários subconjuntos de dados (especialistas, generalistas da ESF e das UBS).

A etapa seguinte, a classificação dos dados, foi operacionalizada pela leitura horizontal e repetida dos textos. Por meio deste "exercício inicial, denominado por alguns autores como leitura flutuante, permite-se apreender as 'estruturas de relevância' dos atores sociais" ${ }^{\prime \prime}$. Nelas estão contidas as ideias centrais dos entrevistados sobre o tema, das quais emergirão as categorias empíricas. No segundo momento, ocorreu a leitura transversal dos dados de cada subconjunto e do conjunto em sua totalidade, que deu origem às áreas temáticas.

A análise final permitiu fazer uma inflexão sobre o material empírico e o analítico, num movimento incessante que se eleva do empírico para o teórico e vice-versa. Esta dança que promove relações entre o teórico e o empírico, o concreto e o abstrato, o geral e o particular, a teoria e a prática é o verdadeiro movimento dialético visando ao concreto pensado ${ }^{10}$. A análise e a discussão dos dados deram-se à luz das evidências científicas recentes em âmbito nacional e internacional.

Foram respeitados os aspectos éticos referentes à pesquisa, conforme a Resolução 466/2012 ${ }^{12}$. A pesquisa foi submetida ao Comitê de Ética credenciado e aprovada por ele. Os participantes foram identificados por letras e números, de forma a preservar o anonimato (PESF1, PESF2, PESF3...).

\section{Resultados e Discussão}

\section{Processo de trabalho na Estratégia Saúde da Família}

O apoio matricial foi descrito como trabalho instituído. Os profissionais referem que, mediante o apoio dos especialistas, atendem às demandas em saúde mental. Os dados coletados evidenciam que o processo de trabalho contempla o atendimento e a intervenção conjunta, com intercâmbios entre a equipe e o apoiador na avaliação do caso e na orientação de condutas, permanecendo o caso sob o cuidado da equipe de referência. Contempla ainda o atendimento ou as intervenções específicas do apoiador, mantendo a equipe de referência o seguimento complementar do cuidado. Essa modalidade é utilizada naquelas situações em que o usuário é referenciado para um serviço de atenção secundária ou terciária.

Os profissionais consideram o AM uma ferramenta importante para o manejo de situações de 
saúde mental na APS: Eu vejo positivamente o AM, com certeza [...] vem qualificando a assistência na APS. As ações que a gente tem vão desde as reuniões com planejamento de processo terapêutico, plano terapêutico e têm também as redes de psicoterapia que saíram também, o (profissional X) já faz há 20 anos, os pacientes podem circular pelas unidades e acompanhar os grupos (PESF2). Outro entrevistado afirmou que com o AM consegue identificar casos que seriam tratados de forma errônea: [...] a bipolaridade que é uma coisa que cada vez mais vem sendo conhecida [...] os médicos tratavam tudo como depressão [...] davam um antidepressivo, e a pessoa ficava mais maníaca, muitas vezes causando a morte do paciente por suicídio (PESF7).

$\mathrm{O}$ atendimento conjunto foi definido como enriquecedor ao aprendizado. É a preferida tanto para o profissional que vivencia essa modalidade, como para os profissionais que experienciam a modalidade de discussão clínica. Entretanto os profissionais relataram que ocorre resistência por parte dos apoiadores na adoção dessa metodologia de trabalho. A forma de apoio relatada com mais frequência é a discussão de casos, mas esta não é considerada uma regra e depende dos arranjos estabelecidos entre o $\mathrm{AM}$ e a equipe de referência. Estudo ${ }^{13}$ evidencia que consultas compartilhadas entre generalistas e especialistas com a presença do paciente produzem conhecimento, compartilhamento de competências entre parceiros de serviços e tratamento coordenado para o paciente. Outros benefícios do contato incluem a melhoria da comunicação, a criação de atitudes positivas e a redução da probabilidade de mal -entendidos e desacordos.

A modalidade de atendimento ou intervenções do apoiador, na qual a equipe de referência faz seguimento complementar do caso ${ }^{14}$ foi definida como geradora de conflitos desde o início da implantação do AM. Observa-se que uma dificuldade do processo reside na integração e na retaguarda do serviço especializado, o CAPS. Em regra, o agendamento é realizado por intermédio do apoiador, o que gera descontentamento pela possível desvalorização da equipe da ESF em sua capacidade de avaliação. Emergiu no GF que não são todos os apoiadores que restringem o acesso ao CAPS. Essa parece ser uma prática dos profissionais não vinculados ao AM. A cogestão e a corresponsabilização são os pilares do AM. Estabelece-se, assim, o eterno tensionamento de quem é o paciente. Cunha e Campos ${ }^{15}$ lembram que o AM é uma metodologia complementar àquela prevista nos sistemas hierarquizados e não substitutiva.
Além dos casos mais prevalentes de transtornos mentais (depressão, transtornos ansiosos), alguns profissionais reportaram também conseguir manejar quadros mais complexos (psicoses, transtornos do humor), e estimam atender em torno de $85 \%$ a $99 \%$ dos casos de saúde mental. Ou seja, há o consenso de que os casos referenciados são efetivamente complexos e demandam o encaminhamento para serviços especializados. Os participantes defendem a manutenção de um sistema de referência para os casos de difícil manejo; refratários ao tratamento; quadros agudos; para pessoas que se beneficiariam de psicoterapia e aqueles que desejam que o atendimento seja feito por um psiquiatra. Nesse sentido, diferentes profissionais abordaram implicações éticas relativas ao acesso ao especialista no reconhecimento da necessidade de uma intervenção especializada.

Os profissionais reconhecem que, com o AM, há a necessidade de se investir em tempo de consulta, assim como de estudar para ter o domínio das situações mais frequentes de saúde mental. Entretanto a instituição de um sistema de referência em saúde mental pressupõe que os profissionais da ESF assumam a responsabilidade pelo paciente, para que somente os casos complicados fossem referenciados. Essa perspectiva é referida na fala: [...] se tu estás apertado com todos os pacientes alguma coisa está errada, tu também vais ter que estudar, vais ter que ver, porque não podem sobrar todos eles no final do mês para o psiquiatra. (PESF 7). O entrevistado reconhece que a referência em algumas situações era utilizada por outros profissionais para a não responsabilização pelo paciente. Nesse caso, a situação posta é como passar de uma lógica de encaminhamento/ desresponsabilização, para a corresponsabilização. Os profissionais reconhecem que o AM, nessa perspectiva, demanda profissionais engajados e comprometidos de ambos os "lados", especialistas e generalistas.

Pode-se depreender que cada apoiador utiliza metodologias e diretrizes de trabalho próprias. Alguns preferem o atendimento conjunto, enquanto outros priorizam a discussão de casos clínicos, sem a presença do usuário. Especialmente no GF, essas diferentes modalidades de atendimentos geraram controvérsias, em razão das USF que têm a segunda modalidade de AM, a discussão de casos clínicos, sentirem-se desfavorecidos com relação aos profissionais das USF onde ocorre o atendimento conjunto. Como alternativa emergiu a possibilidade de rodízio dos apoiadores, de modo que os atualmente "privilegiados" com o atendimento conjunto expe- 
rienciem a modalidade de discussão de casos. Entretanto essa proposta não foi consensual: os profissionais que têm o suporte do atendimento conjunto não querem substituir pela discussão dos casos. Nesta última modalidade, os profissionais sentem-se responsabilizados na gestão do caso, e não corresponsabilizados.

\section{Rede de Atenção à Saúde (RAS) e Rede de Atenção Psicossocial (RAPS)}

Nas falas dos profissionais, evidencia-se disponibilidade para o atendimento no serviço de urgência e emergência pública do município, denominado 24 horas. Em contrapartida, mesmo após a avaliação pelos profissionais das USF, o encaminhamento de algumas situações que não se constituem em urgências, mas sem possibilidades de manejo na unidade, são "devolvidos" pelos CAPS, sob a alegação que existe o AM. Essa situação é expressa por todos os entrevistados. [...] a gente consegue encaminhar com facilidade para o $24 \mathrm{~h}$, por exemplo, um caso agudo, urgente, mas um caso grave que não seja urgente, a gente não consegue [...]; eles usam o matriciamento como uma porta fechada (PESF6). A dificuldade de acesso aos serviços da rede foi também denunciada nos estudos de Morais e Tanaka ${ }^{16}$, o que sugere que a coordenação do cuidado integral, por meio da organização das respostas ao conjunto das necessidades, é um desafio para o SUS. Destaca-se que tanto o acesso geográfico como o organizacional incluem peculiaridades que favorecem ou impedem as pessoas de receber os cuidados de que necessitam ${ }^{17,18}$.

Como já foi dito, mesmo que exista o $\mathrm{AM}$, ainda haverá necessidade de encaminhamentos para serviços de atenção secundária e terciária. Entretanto, para além da estruturação dos serviços, essa situação pode evidenciar características das relações estabelecidas entre os profissionais e destes com a população. Os participantes do estudo avaliam existirem rupturas entre os princípios do SUS e da Reforma Psiquiátrica e o atendimento disponibilizado pelos CAPS, sobretudo no acolhimento transversal (entre equipes) e horizontal (disponibilidade de serviços no curso de um transtorno $)^{19}$.

Os profissionais apontam uma diferença importante: a relação personalizada com os profissionais do AM e não o CAPS, mediante os vínculos estabelecidos, para a discussão de casos que deveriam ser encaminhados para um serviço especializado. [...] estabelecer alguns critérios para que isso funcionasse, que a gente não dependesse apenas desse vínculo só com profissional [...] que existisse essa harmonia [...] (PESF8). Com os vínculos que eu criei com esse psiquiatra e não com o CAPS [...] (PESF8). [...] mas eles (apoiadores) também estão sempre disponíveis para uma troca de informações por contato telefônico (PESF4). Estudo ${ }^{13}$ mostra que problemas decorrentes de acessibilidade pode reduzir a frequência do pedido de ajuda, quando necessário. Por outro lado, o aumento da acessibilidade aos serviços especializados pode levar a mais serviços de consultoria por telefone e menos contato direto de pacientes com profissionais especialistas.

As dificuldades anteriormente referidas com o serviço de atenção secundária são relatadas pelos profissionais. Aqui são as dificuldades de encaminhamento... Ah... nem sei te dizer, eu fico tão triste com isso. É o que dificulta, tu teres um paciente que tu te responsabilizas por ele, como eu falei anteriormente, e tu parares numa porta fechada e dali não passar entendeu? (PESF7). Como afirmam Campos e Domitti ${ }^{14}$, uma lógica de fragmentação do cuidado e de estrutura cria dificuldades gerenciais para a adoção do método de apoio matricial. Nos é passado é que tu não podes encaminhar um paciente via referência $e$ contrarreferência para o CAPS II, e isso na verdade eu acho um erro (PESF3). Dentre os fatores que impedem ou dificultam o acolhimento e o tratamento de pessoas com esquizofrenia na APS, estão a falta de capacitação, de supervisão, de pessoal, de escuta de qualidade, a sobrecarga de trabalho e a ausência de um sistema de referência operante ${ }^{20}$. Os dois últimos fatores também foram relatados pelos profissionais da APS como dificultadores do cuidado.

Apesar de o sistema de referência ser considerado um problema, os profissionais reportam como adequada a contrarreferência do CAPS para as unidades da ESF. Entretanto pontuam esta não ser uma via de mão dupla. Um dos fluxos instituídos é que o paciente em alta - mesmo quando encaminhado diretamente da USF para o serviço de urgência -, deve ser contrarreferenciado para o CAPS e deste para a USF. Os profissionais têm a expectativa de que, com a implantação do prontuário eletrônico, essas questões possam ser otimizadas. O paciente interna lá no 24h, de lá vai para o CAPS e do CAPS vem para cá, e não tem essa ligação direta daqui com o 24h, do 24 para cá, do Hospital com a APS também é bem desvinculado (PESF 2). Observa-se que os fluxos e contrafluxos são burocratizados, ficando o paciente, às vezes, no vácuo, à espera de tratamento.

A fragilidade dos sistemas de informação clínica impede a ESF de introduzir a gestão de base 
populacional e exercitar funções de coordenação das RAS $^{8}$. Esses achados encontram ressonância na pesquisa de Cecílio et al. ${ }^{21}$, que mostra que a APS não reúne condições materiais e simbólicas para funcionar como o centro de comunicação na complexa rede de cuidados. São sugeridos dois movimentos complementares para avançar na constituição, na coordenação e na operacionalização das redes temáticas: a coordenação das redes assumirá múltiplas configurações a depender dos diferentes contextos locorregionais do SUS; os investimentos na APS, para que ela possa ampliar a capacidade de intervir nos processos regulatórios, consolidando-se como centro efetivo de comunicação com os demais serviços.

Os profissionais afirmam que a estruturação dos Núcleos de Saúde da Família (NASF) no município poderia garantir a retaguarda para os casos de saúde mental sem que os CAPS deixassem de ser o serviço de referência de nível secundário. [...] a rede tem que estar mais estruturada, dentro da estratégia tem a questão dos NASF, também que poderia ter psiquiatras que fizessem o AM... (PESF2). Tal posição é corroborada na ideia de que a presença de especialistas em saúde mental na APS, integrados à retaguarda de serviços de atenção secundária é necessária. Para que isso ocorra, o funcionamento adequado do sistema de Atenção Primária é um pré-requisito essencial para a integração dos serviços de saúde mental ${ }^{22}$.

A integração permite a resolução de casos na APS e evidencia outros que necessitam de atenção especializada. O não reconhecimento dessa necessidade traz à tona a insatisfação dos generalistas. Para eles, a descentralização da saúde mental por meio do AM fechou a porta de acesso ao CAPS. Assim, pode-se depreender que há tensionamentos e rupturas na articulação em rede, sobretudo no que concerne ao encaminhamento de pessoas ao CAPS. Os profissionais do CAPS não aceitam a referência direta de um profissional da ESF, sob a justificativa de que existe o AM, sendo necessária a intercessão do apoiador. Em última análise, esses tensionamentos configuram disputas de poder e protagonismo.

\section{Princípios e valores que sustentam o AM}

O termo princípio é designado como o "ponto de partida e fundamento de um processo qualquer". Aos diferentes conceitos sobre princípios formulados o que todos os significados têm em comum é o princípio como ponto de partida do ser, do devir ou do conhecer. Valores podem ser considerados possibilidades de escolha, sempre que determinadas condições se verifiquem, inscrevendo assim valores à universalidade e à permanência ${ }^{23}$.

Foram associadas à questão dos princípios, as noções de interdisciplinaridade; a relação de longitudinalidade que propicia o vínculo; o tempo para a escuta; o sigilo; o respeito; a não dissociação saúde física e mental, a sensibilidade para identificar o sofrimento; a demanda da pessoa; a forma de ajuda. Outras noções citadas foram a unificação da linguagem e o intercâmbio profissional. A integralidade da atenção, com um olhar atento às questões emocionais, também aparece nos relatos dos entrevistados, assim como a resolutividade e a acessibilidade.

A integralidade do cuidado foi reportada pelos profissionais na compreensão de que as questões de ordem emocional e de saúde mental são transversais a todas as práticas. A pertença ao território significa mote para a responsabilização - mesmo com o foco da atenção especializada, a equipe continua articulada com o caso. A longitudinalidade aparece como um atributo que propicia o acompanhamento das situações de doenças e crises da família no curso da vida de seus integrantes. A gente atende toda a família, a clínica é mais ampliada ainda, não só em relação à pessoa, mas em relação à família (PESF1). O olhar contextualizado para o cenário amplo da vida das pessoas e o resgate da história de vida permite a planificação de projetos terapêuticos singulares na perspectiva do paradigma da clínica ampliada e de produção de vida.

$\mathrm{O}$ acolhimento como um princípio profissional foi referido pelos participantes sob diferentes perspectivas. $\mathrm{O}$ acolhimento longitudinal (no tempo) e o acolhimento vertical (a disponibilidade para o cuidado $)^{19}$ foram citados como pontos fortes nas USF. A questão do acolhimento é o início de todo o vínculo. A questão do acesso, o paciente tem que conseguir acessar o sistema, acessar o profissional, acho que também é importante garantir tempo de consulta para o paciente (PESF2). [...] quando a gente consegue ter essa relação mais próxima de pessoa para pessoa, realmente a gente se sente melhor e mais realizado (PESF1). A acessibilidade, a longitudinalidade, a integralidade são princípios e diretrizes do SUS e da Reforma Psiquiátrica e estão contidos nas falas. A integração da saúde mental na APS, sobretudo em cenários com recursos insuficientes, pode contribuir para a acessibilidade e a disponibilidade ${ }^{5}$.

A confidencialidade, mediante o sigilo das informações que o paciente traz, foi considerada um princípio fundamental. Da mesma forma, a 
confiança estabelecida na relação equipe-paciente, equipe ou profissional de referência e AM. Outro aspecto que perpassa a fala de generalistas diz respeito ao interesse de ambos em qualificar a atenção em saúde mental e diminuir o sofrimento. [...] existe um interesse real de que as coisas melhorem na área de saúde mental porque as pessoas que fazem o AM aqui, tanto os que recebem quanto os que nos fornecem, se importam com o sofrimento humano, eles se importam de verdade (PESF7). A construção da autonomia e protagonismo do usuário e a validação do sofrimento aparecem como atributos necessários aos profissionais. [...] tentar que a pessoa se transforme em agente de si mesma (PSF5). Para mim hoje em dia, o que mais me toca, e eu acho que a questão do AM ajuda muito para a gente conseguir abordar essas questões de vida da pessoa, de história de vida, a gente vê que tudo tem uma causa (PESF1).

Nas entrevistas, evidencia-se que a relação do profissional da ESF e do AM, mediante o vínculo estabelecido, promove o enriquecimento das intervenções terapêuticas, sobretudo, quando esse vínculo é baseado em relação de confiança e comprometimento. O vínculo profissional é visto como um valor. É um valor profissional o vínculo profissional, hoje em dia se fala muito no vínculo com o paciente mais não se fala tanto no vínculo da equipe, e eu acho que é tão importante quanto (PESF6). Acho que daí veio o bom relacionamento. De ver nele um profissional que estava ali para me ajudar (PESF4). Esse valor é consensual ao grupo, tanto entre generalistas como entre os especialistas. A relação entre os profissionais sustenta-se fortemente nesse valor e potencializa as intervenções. Além desse, também foram referidos como valores invisíveis importantes o interesse e o envolvimento de ambos, generalistas e especialistas.

De outra forma, pôde-se observar no GF que, quando esse valor foi, de alguma forma, fragilizado ou apresenta tensionamentos, os demais valores que dão sustentação ao processo de trabalho ficam em segundo plano. Ocorre então, um retraimento do profissional da ESF em levar casos para a discussão e planificação de projetos terapêuticos. No GF, emergiram as dificuldades de relacionamento com um dos apoiadores, apontado por dois dos profissionais presentes. Ele é muito sarcástico. Ele usa a culpa e a vergonha no relacionamento dele e isso é muito frustrante, eu estou com muita raiva (PEF2). Entretanto os mesmos profissionais (assim como os demais) reconhecem a excelência técnica do profissional. Percebe-se que as dificuldades são de relacionamento interpessoal, o que dificulta o vínculo, im- pedindo que esses profissionais sintam-se à vontade para solicitar ajuda. $\mathrm{O}$ estresse profissional, relatado pelo aumento da demanda e a pressão por resolver os problemas é intensificado na existência de conflitos entre a equipe. Essas situações foram mencionadas como um obstáculo à realização do trabalho.

A humanidade é referida como um valor essencial. É expressa como a centralização na pessoa atendida, mediante uma relação personalizada, permeada pela sensibilidade, pelo interesse, pela responsabilidade e comprometimento com o paciente. Derivam da humanidade o respeito, a consciência da necessidade de compreensão do paciente e a tranquilidade nas decisões clínicas. A maturidade profissional é vista como um aspecto importante para a personalização da relação e a superação da centralização nos aspectos técnicos, visualizada como uma forma de proteção. A motivação para o atendimento; o interesse; o compromisso com o paciente e com colega (apoiador e outros profissionais da equipe); a disponibilidade para ver e conhecer o paciente; o não julgamento; a centralização na pessoa e não nos seus sintomas foram reportados como valores que perpassam a prática.

A honestidade e a humildade do profissional da ESF em receber orientação de um especialista emergem como valores indispensáveis ao AM. A humildade é reconhecida como tarefa difícil na relação entre profissionais da área médica, pois implica o reconhecimento de que não há domínio daquela área. De outro lado, os apoiadores também necessitam ter uma postura de humildade na inter-relação com a equipe de referência. Esta é vista como um fator que permite a abertura, ao expor-se. Honestidade, disciplina, humildade, criatividade e curiosidade foram identificadas ${ }^{24}$ como valores necessários ao trabalho das equipes de saúde. Dentre os princípios, estão o compartilhamento de objetivos, papéis claros, confiança mútua, comunicação efetiva, avaliação de processos e resultados. Esses atributos também foram elencados pelos participantes do presente estudo.

A diminuição do estigma que as pessoas com um transtorno mental carregam foi discutida no GF como um valor a ser perseguido. Esse assunto foi introduzido na questão da integralidade do cuidado e não dissociação sofrimento físico e mental. Nesse sentido, a partir do AM na Atenção Primária e do atendimento do generalista, há o entendimento de que este passa ser atenuado. Nesse sentido, o AM e a descentralização desmitifica um pouco a questão do psiquiatra como o último elemento, a pessoa responsável pela saúde mental... 
(PESF2). Eles se sentem mais à vontade, às vezes por não estar com o psiquiatra, que já tem aquele estigma (PESF1). Entretanto os entrevistados mencionam também o estigma dos profissionais generalistas quanto ao atendimento de pessoas com um transtorno mental, que necessita ser superado, além do autoestigma e o estigma da sociedade. [...] eu acho que o médico de família é ensinado a perceber que um sintoma nunca é sintoma só físico [...] porque tem pessoas que já recebem mal porque é a louca, a deprimida [...] são pessoas que não tem essa disponibilidade, essa abertura e nem essa visão de enxergar que tudo é a mesma coisa [...] o corpo não está separado do cérebro (PESF7).

O vínculo profissional como valor foi consenso no GF. A relação entre as equipes sustenta-se nesse valor e potencializa as intervenções. Pôdese observar no GF que, quando esse aspecto foi fragilizado, por questões relacionadas a conflitos de personalidade ou a não concordância com a forma de trabalho instituída (diferentes modalidades de apoio e abordagens ao usuário), resultando em tensionamentos, os demais valores que dão sustentação ao processo de trabalho ficam em segundo plano. Aparece, aí, um investimento de tempo e energia para a solução do conflito, perdendo-se o foco no trabalho terapêutico. A diminuição do estigma (autoestigma, dos profissionais e da sociedade) a que pessoas com transtornos mentais estão sujeitas emergiu como um valor a ser conquistado.

\section{Os fatores que dificultam o AM}

No GF, apesar dos diferentes temas elencados para a discussão, os profissionais começaram pelas dificuldades de encaminhamento para o CAPS. Essas se constituem barreiras percebidas, quando há necessidade de referenciar um paciente ao serviço especializado, após tentativas de manejo na unidade de saúde. Os profissionais reconhecem que o paciente "pertence" à área geográfica do posto, que não se trata de desresponsabilização. Porém expressaram a necessidade de apoio e de reconhecimento na avaliação e conduta específica (avaliação psiquiátrica de casos complexos e psicoterapia).

Os participantes do GF discutiram as especificidades de cada posto para a operacionalização do AM. De acordo com os apoiadores, configuram-se diferentes arranjos organizacionais. Há evidente preferência pelo atendimento conjunto, relatado e vivenciado por somente um dos médicos presentes. Os demais profissionais relataram que a forma de apoio é por meio de discussão de casos clínicos, reservando-se o atendimento conjunto para alguns casos específicos. No atendimento conjunto, foi questionada a participação em alguns casos de vários profissionais, o que gera desconforto para a pessoa que necessita expor a sua vida para outras com as quais não tem vínculo. No GF, com os generalistas, emergiu a questão da necessidade de novos arranjos e a reavaliação da forma de trabalho.

Em regra, há uma dificuldade de encaminhamento para o serviço terciário, quando se faz necessária a internação. Essa situação não se configura na pesquisa. Isso talvez decorra em razão de um dos apoiadores estar alocado também no serviço de urgência. Assim, pelo vínculo estabelecido, os profissionais conseguem o contato, a avaliação e a internação. [...] a gente consegue encaminhar com facilidade para o 24h, por exemplo, um caso agudo, importante, urgente, mas um caso grave que não seja urgente, a gente não consegue encaminhar para o CAPS, e eles sempre usam o matriciamento como uma porta fechada (PESF6). O processo incipiente de interação com os outros níveis de atenção, com potencial de promover a reorganização dos serviços e das práticas, assim como os conflitos inerentes aos serviços e ações foi reportado em outro estudo ${ }^{12}$.

Outra dificuldade abordada pelos profissionais diz respeito à compreensão de o AM não ser uma estratégica da gestão. Não é uma estratégia de gestão o matriciamento. Não, o matriciamento é uma estratégia do Apoiador X. Que foi conquistada por ele. (GF PSF2). [...] veio de baixo para cima, a gestão acabou aceitando isso com o andar da carruagem. (GF PESF4). Outro profissional demonstra surpresa com o comentário: Sempre me pareceu que era uma estratégia coletiva do CAPS, aquela coisa bem maligna, de se livrar dos pacientes (GF PESF4). Eu acho que acabou sendo aproveitado como, foi encampado como a ideia milagrosa, não era para ser assim (GF PSF2). As falas evidenciam diferentes perspectivas sobre o AM, denotando a ausência de diretrizes construídas conjuntamente entre especialistas, generalistas e gestores. A compreensão de que o AM não é uma estratégia da gestão, encontra ressonância também nas falas dos apoiadores. Nas entrevistas e GF com esse grupo, foi evidenciado o esforço empreendido para manter o AM.

O apoio é visualizado, na perspectiva dos profissionais da ESF, como uma forma de resolver a demanda e barrar o acesso aos serviços especializados, principalmente, como uma forma de resolver a demanda do CAPS. Nesse sentido, emergiu a necessidade de investimento na estru- 
tura do CAPS e a contratação de profissionais especializados para fazer frente às demandas crescentes na área. Pesquisa ${ }^{25}$ realizada com o objetivo de identificar a opinião de clínicos gerais sobre o atendimento de saúde mental na APS traz a necessidade de incrementar a capacidade da atenção secundária; otimizar a colaboração entre a atenção primária e a secundária; permitir um tempo adequado para a consulta de pessoas com transtornos mentais na APS. Esses resultados são convergentes com os achados da pesquisa e encontram ressonância na literatura nacional e internacional.

Há o reconhecimento de que essa forma de trabalho é resolutiva, porque capacita as equipes para as intervenções no campo da saúde mental no território. Ou seja, um dos fatores que dificultam o AM diz respeito às diferentes perspectivas e entendimentos sobre o processo. Outra questão, que reflete a anterior, diz respeito aos apoiadores serem vistos a serviço da gestão, no sentido de resolver a demanda. Entretanto, ao cruzar os dados, observa-se que existem, também por parte dos apoiadores, dificuldades de reconhecimento da gestão. Assim, ambos os grupos queixam-se da gestão, por diferentes razões. Essa situação configura a inexistência de diretrizes claras, solicitadas por todos os grupos, apoiadores, profissionais das UFS e UBS. Nesse sentido, foi mencionada a necessidade de participação e posicionamento da gestão no AM, com vista ao estabelecimento de diretrizes e fluxos. [...] diretrizes claras de como é que o AM deve funcionar. A gente não tem essas diretrizes (GF PESF4). Pesquisa ${ }^{26}$ evidencia que as intervenções conjuntas requerem a atenção a vários níveis (do organizacional à gestão da clínica) e o redesenho dos serviços.

As diferentes formas de realização do AM polarizam a discussão no GF. Aqui cabe comentar um viés da pesquisa: dos seis profissionais presentes, somente um vivencia a modalidade de atendimento conjunto. Os demais estão habituados à discussão de casos, com a presença do paciente em situações apontadas como "excepcionais". O atendimento conjunto é visto pelo grupo como a modalidade desejável. Nesse sentido, para que sejam equalizadas as oportunidades de vivenciar diferentes apoiadores e formas de AM, emergiu do GF o desejo pelo rodízio de apoiadores. Entretanto, um profissional, que vivencia o que denomina de "casamento perfeito" com o apoiador, não se mostrou favorável a tal modificação.

A entrada de profissionais na ESF via PROVAB é visto como um fator dificultador que impede a relação longitudinal com a comunidade. A rotati- vidade de profissionais (turnover) é mencionada em outro estudo brasileiro como uma barreira à colaboração entre profissionais da APS e de saúde mental ${ }^{27}$. Outro aspecto dificultador relatado pelos médicos diz respeito à necessidade de assumir limitações e ter humildade para o reconhecimento destas, de forma bilateral. Acho que a dificuldade é essa mesmo, é uma questão de humildade de ambas as partes e de também reconhecer as limitações [...] (PESF1). Voltar a ter a possibilidade de fazer referência para o CAPS dos casos selecionados, não ter essa restrição absurda de não encaminhar para o CAPS (PESF5). Matriciamento é um apoio, não uma substituição de um serviço (PESF2). Os profissionais queixaram-se de que o julgamento clínico da necessidade de acompanhamento de casos complicados não é valorizado na referência ao CAPS. Usualmente o agendamento de uma avaliação no CAPS é realizado por intermédio do apoiador. Essa questão foi abordada em diferentes momentos, tanto nas entrevistas, como nos GF. Nesse sentido, a reavaliação dos papéis de generalistas e especialistas é um requisito essencial ao cuidado compartilhado ${ }^{26}$.

\section{Os fatores que facilitam o AM}

Os fatores que facilitam a integração da saúde mental na APS por meio do AM podem ser divididos em estruturais, organizacionais, tecnológicos e relacionais. Os últimos desempenham um papel central nos arranjos realizados entre profissionais generalistas e apoiadores. Pode-se depreender que as tecnologias leves dão sustentação à prática instituída. $\mathrm{O}$ vínculo com o profissional que realiza o AM é relatado como um fator que facilita o trabalho intersetorial e interdisciplinar. [...] esses vínculos vão se estreitando e esses vínculos é que promovem e facilitaram que se estabelecesse o suporte do matriciamento com este profissional (PSF8).

A estrutura das equipes da ESF foi citada como um fator que promove o trabalho interdisciplinar. Também foi mencionada a iniciativa dos apoiadores em realizar reuniões do AM em diferentes locais da rede, como, por exemplo, o CAPSad, a Casa do Idoso, a farmácia municipal e nos CAPS. Essas ações promovem o intercâmbio com outros serviços da rede e são consideradas positivas. O compartilhamento de experiências e de saberes por meio das interações estabelecidas com os colegas de diferentes unidades de saúde propicia a troca de informações, as mudanças de pontos de vista e a incorporação de diferentes perspectivas na instituição de planos terapêuti- 
cos. Também isso ocorre no compartilhamento de angústias e no apoio.

Dentre os fatores que promovem o trabalho intersetorial, foram mencionados o próprio AM em saúde mental, o acesso ao serviço de urgência e a comunicação. A relação personalizada com os profissionais é citada como fator facilitador. A longitudinalidade é referida tanto na relação com o paciente, como também com o colega. [...] é eu acho que é a questão da longitudinalidade de novo, porque daí a gente conhece as pessoas, a gente não trata simplesmente 'ah é o 24 horas', não, tu ligas para lá e falas com o fulano, falas com o cicrano, então tu conheces as pessoas, então tu sabes quem é que esta lá, quem é o neurologista, quem é o psiquiatra (PESF 2).

Pode-se depreender que a relação personalizada com os apoiadores, o vínculo, a possibilidade de contato, referida como o acesso e a disponibilidade do apoiador, e a comunicação fluida dão sustentação à prática do AM. [...] o que promoveu foi a proximidade que eu tenho com ele (apoiador) pelo tempo que a gente trabalha aqui [...] a gente está trabalhando juntos há seis anos [...] esses vínculos vão se estreitando e esses vínculos é que promovem e facilitaram que se estabelece esse suporte do AM com este profissional (PSF8). Essa relação demanda a construção conjunta, o querer fazer e o respeito aos estilos de trabalho. Da mesma forma, isso acontece, quando ambos - especialistas e generalistas se empenham na construção e na solidificação de relações ancoradas na corresponsabilização. A sistematicidade dos encontros foi mencionada tanto pelos apoiadores como pelos generalistas como uma necessidade longitudinal. Assim, uma questão que deve ser considerada na implantação de uma prática como o AM diz respeito à previsão de recursos humanos (especialistas) que possam ser alocados para tal finalidade.

Pesquisas avaliativas quanto à efetividade do AM na APS ainda são escassas, em razão de essa metodologia ainda ser relativamente recente e não adotada de maneira uniforme por estados e municípios. Pesquisa ${ }^{27}$ realizada em quatro cidades (São Paulo, Rio de Janeiro, Porto Alegre e Fortaleza) objetivando avaliar a efetividade de intervenções em saúde mental, antes e após capacitações em saúde mental de 96 horas para médicos e enfermeiros generalistas da APS para o cuidado compartilhado, concluiu que a capacitação dos profissionais não está associada de forma consistente com o reconhecimento ou a gestão de problemas de saúde mental. Os resultados do estudo evidenciam que os enfermeiros aumentaram as taxas de reconhecimento (de
$23 \%$ para $39 \%$ ), enquanto as taxas dos médicos generalistas diminuíram (de $42 \%$ para $30 \%$ ). Assim, embora algum impacto tenha sido observado em enfermeiros, as mudanças no sistema de cuidado requerem o incremento na formação, nas mudanças de comportamento e nos modelos de educação específicos.

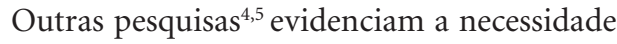
de supervisão e suporte de forma sistemática de profissionais especialistas para os generalistas. As pesquisas disponíveis permitem fazer algumas inferências, como, por exemplo, a necessidade de suporte aos profissionais generalistas por meio de acompanhamento sistemático longitudinal por especialistas. A perspectiva em questão encontra ressonância nos dados empíricos e diz respeito à necessidade de esse suporte e apoio por parte dos especialistas ser longitudinal e não ser por meio de intervenções focais pontuais ou esporádicas. Assim, essa pode ser considerada uma diretriz importante no processo, que necessita ser considerada na instituição dessa metodologia pelos profissionais e gestores.

A preocupação que emergiu dos participantes do GF diz respeito à questão da aposentadoria de alguns apoiadores. Existe o entendimento de que há um perfil profissional para ser apoiador. Assim, um concurso público não poderia aferir as características necessárias ao devir apoiador: gostar da APS, socializar o conhecimento, ter humildade e disponibilidade para considerar posições diferentes, circular em diferentes territórios, além de ser receptivo ao inusitado. Guardadas as devidas singularidades, porque falamos de pessoas, e destas em interação com outras, essas parecem ser algumas das características presentes nos apoiadores, objeto de outra análise.

\section{Considerações Finais}

O AM parece como uma prática instituída, sustentada por meio das relações personalizadas entre os profissionais e os apoiadores. Há o consenso entre os profissionais que o AM qualifica as intervenções em saúde mental. Entretanto, os tensionamentos ocorrem à medida que o AM é utilizado como uma maneira de impedir o acesso aos serviços de atenção secundária, particularmente ao CAPS. As modalidades utilizadas com mais frequência são as discussões de projetos terapêuticos e os atendimentos conjuntos. Esses arranjos estão na dependência de como cada apoiador institui o seu modo de trabalho. A prática clínica está ancorada na perspectiva da am- 
pliação da clínica, no paradigma psicossocial e de produção de vida. Há a noção de "cura" como um processo singular do sujeito, mediado pelos profissionais.

Os princípios profissionais que dão sustentação às práticas são a interdisciplinaridade, $\mathrm{o}$ acolhimento, o vínculo, a integralidade do cuidado, a resolutividade, a acessibilidade e a longitudinalidade. Esses princípios encontram ressonância nos princípios e nas diretrizes do SUS e na Reforma Psiquiátrica brasileira. Outros incluem a qualificação profissional, a confidencialidade e o interesse em qualificar a atenção em saúde mental. Os valores foram relacionados aos usuários e à interação com o apoiador. Dentre os primeiros, estão a humanização e a motivação para o atendimento, o respeito, o vínculo e a centralização na pessoa e não nos sintomas. Os valores que sustentam a relação com o apoiador organizam-se em torno do vínculo, da confiança, da honestidade e da humildade. O vínculo com o especialista, por meio de uma relação personalizada, a estrutura das equipes da ESF e a troca de experiências e saberes aparecem como fatores facilitadores. As dificuldades de encaminhamento ao CAPS, a ausência de diretrizes claras, construídas conjuntamente e a ausência de reconhecimento dos gestores emergem como fatores dificultadores.

Da reinterpretação dos dados pode-se inferir que o AM mostra potência de transformação de saberes e práticas. Do mesmo modo, possibilita novos agenciamentos e processos de trabalho no território. Pode-se depreender que, para além das dificuldades de ordem política e estrutural, o AM mantém-se em razão das relações personalizadas e longitudinais que foram construídas e consolidadas no campo de interação. Há o consenso entre os profissionais de que o processo é eminentemente profissional dependente, construído por meio de tecnologias leves. Na realidade estudada, uma fragilidade que merece ser discutida diz respeito à necessidade da gestão de incorporar essa metodologia de trabalho, mediante a sustentação de apoiadores e generalistas. Isso se deve ao fato de o AM ter sido instituído não por um imperativo da gestão, mas do comprometimento de um grupo de especialistas em descentralizar e qualificar a atenção em saúde mental na APS. Estas questões, que não foram objeto do estudo, podem ser apontadas como limitações e se configurar como desdobramentos de novas pesquisas. Outra limitação da pesquisa foi a impossibilidade da realização de um segundo GF com os diferentes grupos participantes. A proposta foi realizada nos três GF, porém, em razão de questões operacionais como a necessidade de cancelamento de agendas e a disponibilidade de um horário comum aos participantes, foi solicitada a ampliação do horário dos GF e a não realização de um segundo encontro. 
Referências

1. World Health Organization (WHO). Mental Health Action Plan 2013-2020. Geneva: WHO; 2013.

2. World Health Organization (WHO). Mental health and development: targeting people with mental health conditions as a vulnerable group. Geneva: WHO; 2010.

3. Ngo VK, Rubinstein A, Ganju V, Kanellis P, Loza N, Rabadan-Diehl C, Daar AS. Grand Challenges: Integrating Mental Health Care into the Non-Communicable Disease Agenda. PLoS Med 2013 10(5):e1001443.

4. Patel V, Maj M, Flisher AJ. Reducing the treatment gap for mental disorders: a WPA survey. World Psychiatry 2010; 9(3):169-176.

5. Minoletti A, Rojas G, Horvitz-Lennon M. Salud mental em atención primaria en Chile: aprendizajes para Latinoamérica. Cad. saúde colet. 2012; 20(4):440-447.

6. Onocko Campos R, Gama CA, Ferrer AL, Santos DVD, Stefanello S, Trapé TL, Porto K. Saúde mental na atenção primária à saúde: um estudo avaliativo em uma grande cidade brasileira. Cien Saude Colet. 2011; 16(2):4643-4652.

7. Campos GWS. Saúde Paidéia. 2a ed. São Paulo: Hucitec; 2003.

8. Mendes EV. O cuidado das condições crônicas na atenção primária à saúde: o imperativo da consolidação da estratégia da saúde da família. Brasília: Organização Panamericana da Saúde; 2012.

9. Brasil. Ministério da Saúde. Portaria 154, de 24 de janeiro de 2008. Dispõe sobre a criação dos Núcleos de Apoio à Saúde da Família. Diário Oficial da União 2008; 25 jan.

10. Minayo MCS. O desafio do conhecimento: pesquisa qua litativa em saúde. 12a ed. São Paulo, Rio de Janeiro: $\mathrm{Hu}$ citec, Abrasco; 2010.

11. Barbour R. Grupos Focais. Porto Alegre: Artmed; 2009.

12. Brasil. Conselho Nacional de Saúde. Resolução no 466 de 12 de dezembro de 2012. Diretrizes e normas regulamentadoras de pesquisa envolvendo seres humanos. Diário Oficial da União 2013; 13 jun.

13. Fredheim T, Danbolt LJ, Haavet OR, Kjønsberg K, Lien L. Collaboration between general practitioners and mental health care professionals: a qualitative study. Int J Ment Health Syst 2011; 5(1):13.

14. Campos GWS, Domitti AC. Apoio matricial e equipe de referência: uma metodologia para gestão do trabalho interdisciplinar em saúde. Cad Saude Publica 2007; 23(2):399-407.

15. Cunha GT, Campos GWS. Apoio matricial e atenção primária em saúde. Saúde Soc 2011; 20(4):961-970.

16. Morais APP, Tanaka OY. Apoio matricial em saúde mental: alcances e limites na atenção básica. Saúde Soc 2012; 21(1):161-170.
17. Brasil. Conselho Nacional de Secretários de Saúde (CONASS). Atenção Primária e Promoção da Saúde. Brasília: CONASS; 2007.

18. Quinderé PHD, Jorge MSB, Nogueira MSL, Costa LFA Vasconcelos MGF. Acessibilidade e resolubilidade da assistência em saúde mental: a experiência do apoio matricial. Cien Saude Colet 2013; 18(7):2157-2166.

19. Thornicroft G, Tansella M. The mental health matrix. A manual to improve services. New York: Cambridge University Press; 1999.

20. Almeida GH. Acolhimento e tratamento de portadores de esquizofrenia na atenção básica: a visão de gestores, terapeutas, familiares e pacientes [tese]. São Paulo: Faculdade de Saúde Pública; 2010.

21. Cecilio LCO, Andreazza R, Carapinheiro G, Araújo EC, Oliveira LA, Andrade MGG, Meneses CS, Pinto NRS, Reis DO, Santiago S, Souza ALM, Spedo SM. A Atenção Básica à Saúde e a construção das redes temáticas de saúde: qual pode ser o seu papel? Cien Saude Colet 2012; 17(11):2893-2902.

22. World Health Organization (WHO), World Organization of Family Doctors (WONCA). Integrating mental health into primary care: a global perspective. Geneva, Bangkok: WHO, WONCA; 2008.

23. Abbagnano N. Dicionário de Filosofia. $5^{\mathrm{a}}$ ed. São Paulo: Martins Fontes; 2007.

24. Mitchell P, Wynia M, Golden R, McNellis B, Okun S, Webb CE, Rohrbach V, Von KohornI. Core principles \& values of effective team-based health care. Discussion $\mathrm{Pa}$ per, Institute of Medicine, Washington, DC. 2012 [cited 2014 Jul 17]. Available from: www.iom.edu/tbc.

25. Mykletun A, Knudsen AK, Tangen T, Øverland S. General practitioners' opinions on how to improve treatment of mental disorders in primary health care. Interviews with one hundred Norwegian general practitioners. BMC Health Serv Res 2010; 10:35.

26. Kelly BJ, Perkins DA, Fuller JD, Parker SM. Shared care in mental illness: A rapid review to inform implementation. Int J Ment Health Sys 2011; 5:31.

27. Goncalves DA, Fortes S, Campos M, Ballester D, Portugal FB, Tófoli LF, Gask L, Mari JJ, Bower P. Evaluation of a mental health training intervention for multidisciplinary teams in primary care in Brazil: a pre-and posttest study. Gen Hosp Psychiatry 2013; 35(3):304-308.

Artigo apresentado em 03/08/2014

Aprovado em 05/10/2014

Versão final apresentada em 08/10/2014 